\title{
Gestión del tiempo y procrastinación en la educación superior
}

\section{Time Management and Procrastination in High Education}

Recepción: 31 Julio 2015 | Aprobación: 28 Febrero 2017

\author{
Angélica Garzón Umerenkova ${ }^{\mathrm{a}}$ \\ Universidad El Bosque, Colombia \\ ORCID: http://orcid.org/0000-0002-6813-552 \\ JaVier Gil Flores \\ Universidad de Sevilla, España
}

\begin{abstract}
a Autor de correspondencia. Correo electrónico: garzonangelica@unbosque.com

Para citar este artículo: Garzón Umerenkova, A., \& Gil Flores, J. (2017). Gestión del tiempo y procrastinación en la educación superior. Universitas Psychologica, 16(3), 1-13. https:// doi.org/10.11144/Javeriana.upsy 16-3.gtpe
\end{abstract}

\section{RESUMEN}

La procrastinación constituye un fallo de las conductas autorregulatorias que conlleva efectos negativos a nivel académico. Analizamos el valor de la gestión del tiempo como variable predictora de la procrastinación, estudiando una muestra de 494 estudiantes universitarios colombianos de primer año, que fueron clasificados en dos grupos en función de su grado de procrastinación académica. Hemos recogido información sobre sus conductas de gestión del tiempo, medidas a través de una versión en español del Time Management Behavior Questionnaire (TMBQ). Tras construir un modelo de regresión logística, las puntuaciones en la subescala "establecimiento de objetivos y prioridades" junto con "percepción sobre el control del tiempo" resultan ser buenas predictoras del nivel de procrastinación, a diferencia de Herramientas para la gestión del tiempo o preferencias por la desorganización. Entre las características demográficas (sexo, edad y dedicación), juega un papel relevante la condición de estudiante con dedicación compartida entre estudio y trabajo.

Palabras clave

procrastinación; gestión del tiempo; educación superior

\begin{abstract}
Procrastination constitutes a self-regulatory failure which conveys many negative effects at an academic level. We analyze time managing values as a predictor of procrastination, studying a sample of 494 Colombian students of first year of University. We gathered information about the frequency with which they postpone tasks and about their time management behaviors using the Time Management Behavior Questionnaire (TMBQ). After building a logistic regression model, the "establishment of objectives and priorities" scale's scores, added to those for perception of control over time, proved to be accurate predictors. This is not the case for time management tools or preferences for disorganization. Among demographics (sex, age and dedication), the condition of students who have a shared dedication to job and studies plays a relevant role.

Keywords

procrastination; time management; higher education
\end{abstract}


La procrastinación es la tendencia a gastar el tiempo, demorar y aplazar de forma intencionada algo que debe ser hecho (Tuckman, 2003), aun siendo consciente de las consecuencias negativas que ello pueda comportar (Klingsieck, 2013). Esta conducta implica posponer el inicio de una tarea, completarla en el último momento, sobrepasar los límites temporales fijados para la misma o incluso evitar indefinidamente su realización. Para ello, el individuo procrastinador adopta un papel activo, decidiendo realizar determinadas tareas antes que las que debería afrontar, frecuentemente por las compensaciones que obtiene o por eludir experiencias aversivas (Rozental \& Carlbring, 2014).

Aunque en este trabajo nos centraremos en el estudio de las conductas de procrastinación de los estudiantes en la educación superior, tales conductas no son exclusivas de este nivel educativo ni del ámbito académico, sino que pueden presentarse en múltiples áreas de la vida de las personas. Entre otros comportamientos, los procrastinadores tienden a no cumplir con sus agendas cuando abordan proyectos personales (Lay, 1990), posponen la visita al médico, con importantes consecuencias para su salud (Bogg \& Roberts, 2004) o acaban realizando sus compras de Navidad ese mismo día (Ferrari, 1993). En algunas personas, las demoras o aplazamientos pueden llegar a ser una característica habitual de su forma de actuar.

Si bien la procrastinación afecta de manera crónica a entre un $15 \%$ y $20 \%$ de la población adulta (Harriott \& Ferrari, 1996), resulta endémica en el ámbito académico. Entre los estudiantes de secundaria se estima una prevalencia del $80 \%$ al $95 \%$ (Steel, 2007), y es un fenómeno corriente en el $70 \%$ de los estudiantes universitarios anglosajones (Ferrari, O'Callaghan, \& Newbegin, 2005). La procrastinación les lleva a retrasar el estudio para los exámenes y a dedicar menos horas de las necesarias (Lay \& Burns, 1991), vinculándose significativamente a un menor rendimiento académico (Van Eerde, 2003; Kim \& Seo, 2015). La procrastinación académica juega un papel importante en la deserción universitaria (Garzón
\& Gil, 2017a), la salud física y mental de los estudiantes o el estrés (Cardona, 2015).

Uno de los aspectos en que se ha focalizado la investigación sobre la procrastinación es su relación con otros rasgos o conductas del sujeto. En el estudio meta-analítico de Steel (2007), se constataron mayores niveles de procrastinación, por ejemplo, entre quienes presentan niveles bajos de autoeficacia, y niveles altos de perfeccionismo, miedo al fracaso, depresión o impulsividad. En cambio, no se han encontrado resultados consistentes sobre la relación de la procrastinación con la extraversión o la inteligencia. En lo que respecta a las características demográficas, este autor apunta a una disminución de la procrastinación con la edad, al tiempo que señala la existencia de niveles de procrastinación ligeramente mayores entre hombres que entre mujeres. En el ámbito universitario, trabajos recientes han confirmado una mayor procrastinación de los alumnos frente a las alumnas (Cardona, 2015; Carranza \& Ramírez, 2013). En el caso del alumnado colombiano, la dedicación del estudiante podría ser otra variable demográfica por considerar en el estudio de la procrastinación. En las universidades colombianas, es frecuente la presencia de alumnado que financia sus estudios universitarios simultaneándolos con actividades laborales. Al analizar este tipo de alumnado no tradicional, Robotham (2012) señalaba que quienes combinan trabajo y estudio consideran un inconveniente la menor disponibilidad de tiempo para realizar las tareas académicas, lo cual podría llevar a conductas procrastinadoras.

Definiendo la procrastinación como la demora voluntaria en el curso de una acción, se puede asumir que esta variable está fuertemente relacionada con los procesos de autorregulación y se entiende como un fallo en la autorregulación del individuo (Pychyl \& Flett, 2012; Rozental \& Carlbring, 2014; Steel, 2007). Desde esta perspectiva, la procrastinación sería atenuada con la organización, la capacidad de establecer metas y estructurar las propias tareas, la motivación hacia el rendimiento o la disposición a cubrir los planes de trabajo. Las tareas que son percibidas como difíciles, poco atractivas, 
ambiguas y que requieren más esfuerzo que otras presentan mayor probabilidad de ser objeto de procrastinación (Ferrari, Mason, \& Hammer, 2006).

El presente trabajo se ha centrado en el análisis de la relación entre la gestión del tiempo y la procrastinación. La gestión del tiempo constituye un elemento crítico en el trabajo académico del alumnado universitario, resultando ser el factor personal y académico más importante en la predicción del rendimiento, por delante de otros tales como la inteligencia o el tiempo dedicado al estudio (George, Dixon, Stansal, Lund, \& Pheri, 2008). Estudiando al alumnado universitario, Durán-Aponte y Pujol (2012) encontraron correlaciones significativas entre el rendimiento y dimensiones de la gestión del tiempo, tales como el establecimiento de objetivos o el uso de herramientas de gestión. Además, se ha hallado superioridad de las alumnas sobre los alumnos en lo que respecta a la gestión el tiempo (DuránAponte \& Pujol, 2013), y relaciones significativas entre una buena gestión del tiempo y el desarrollo del carácter, entendido como adopción de valores éticos (Olowookere, Alao, Odukoya, Adekeye, \& Agbude, 2015), la satisfacción con la vida (Krumrei, Elizabeth, Newton, Kim, \& Wilcox, 2013) o la reducción de la ansiedad y buen manejo de las relaciones sociales y recreativas (Kaya, Kaya, Ozturk, \& Kucuk, 2012).

La gestión del tiempo académico constituye un componente clave de la autorregulación del aprendizaje (Barber, Munz, Bagsby, \& Grawitch, 2009; Pintrich, Smith, García, \& McKeachie, 1993; Zimmerman, 1996), relacionado con otros factores cognitivos (p. ej., autoevaluación y automonitoreo) y motivacionales (p. ej., autoeficacia y creencias de control sobre el aprendizaje) presentes en la autorregulación académica. Sin embargo, no existe una definición unificada sobre el constructo gestión del tiempo. Como señalan Liu, Rijmen, MacCann y Roberts (2009), la gestión del tiempo se ha definido y operacionalizado para fines investigadores de muy variadas formas, aunque la mayor parte de ellas converge en una idea central: la finalización de tareas o actividades en un tiempo esperado, obteniendo resultados de calidad, mediante procedimientos tales como la planificación, la organización o la priorización. En esta misma línea, Claessens, Van Eerde, Rutte y Roe (2007), tras la revisión de 34 estudios empíricos sobre manejo del tiempo desde 1982 hasta 2004, concluyeron que la gestión del tiempo se refiere a conductas que buscan lograr un efectivo uso del tiempo, mientras se ejecutan ciertas actividades dirigidas a una meta, por lo que se trata de un proceso dinámico relacionado con aspectos estratégicos y motivacionales del aprendizaje. La gestión del tiempo académico está dirigida por el establecimiento y logro de metas, al mismo tiempo que implica una supervisión y regulación de las metas establecidas. Por tanto, dentro del modelo de autorregulación académica, la gestión del tiempo tiene un fuerte componente motivacional y de autocontrol.

Teniendo en cuenta que la procrastinación implica un déficit de conductas autorregulatorias en general, cabe esperar que lo sujetos procrastinadores presenten un bajo nivel en las conductas relativas a gestión del tiempo. No obstante, aunque los constructos procrastinación y gestión del tiempo pueden ser enmarcados en las conductas autorregulatorias, con el tiempo como elemento común a ambos, es escasa la literatura que aborda de manera conjunta la investigación de ambos conceptos.

El presente estudio trata de responder a esta carencia, atendiendo además a la necesidad de nuevas investigaciones señalada por Steel (2007). Tras su metaanálisis sobre casi setecientos estudios, este autor concluía que la procrastinación representa un fallo en la autorregulación que aún no se ha explicado suficientemente $y$, dado el crecimiento de su prevalencia, consideraba necesario proseguir con la investigación sobre este tema. Por otra parte, la gestión del tiempo es generalmente conceptualizada como un conjunto de conductas dirigidas a facilitar la realización de tareas. Macan, Shahani, Dipboye y Phillips (1990) señalaron el carácter multidimensional de este constructo, en el que cabe identificar factores independientes. Asumiendo estos planteamientos, el presente trabajo se centra en la explicación de las 
conductas procrastinadoras en la educación superior. Concretamente, su objetivo es analizar la importancia diferencial de los distintos factores de gestión del tiempo en la explicación de la procrastinación académica, controlando el efecto atribuido a las características demográficas de los estudiantes.

\section{Método}

\section{Participantes}

Los participantes en el estudio fueron 494 estudiantes de primer año, matriculados en la Universidades El Bosque y Uniempresarial, ambas de la ciudad de Bogotá. La muestra fue seleccionada en función de criterios de accesibilidad, y se aseguró la heterogeneidad de la misma al incluir estudiantes de las titulaciones de Psicología, Educación Bilingüe, Pedagogía, Ingeniería de Sistemas, Ingeniería Electrónica, Administración de Empresas, Finanzas y Comercio Exterior, Negocios Internacionales y Marketing. Del total de estudiantes considerados, 367 eran hombres y 127 mujeres, con edades comprendidas entre los 16 y 46 años y con una media de 19.31 años $(D E=3.56)$; el $55.6 \%$ eran estudiantes con dedicación exclusiva y el $44.4 \%$ comparten el estudio con una actividad laboral.

\section{Variables e instrumentos}

Hemos tomado como variable dependiente el nivel de procrastinación. Se trata de una variable binaria (niveles alto/bajo), construida tras clasificar al alumnado universitario en función de sus conductas procrastinadoras. Estas conductas fueron medidas utilizando ítems incluidos en la primera sección del PASS (Procrastination Assessment Scale-Students), creado por Solomon y Rothblum (1984). Se aplicó la versión en español de este instrumento validada para población universitaria colombiana por Garzón y Gil (2017b), que presentó para el primer atributo un buen ajuste al modelo Rasch con valores para todos los ítems entre 0.5 y 1.5
(MNSQ infit y outfit). Además, estos autores señalan valores de confiabilidad de 0.99 para los ítems y 0.86 para los sujetos, y establecen evidencias de validez discriminante y predictiva, obteniendo correlaciones negativas significativas frente a las habilidades de gestión del tiempo y el promedio de notas.

Concretamente, para este estudio, se utilizaron los seis elementos referidos a la frecuencia con que los estudiantes posponen otras tantas tareas: escribir un trabajo de final de curso, estudiar para los exámenes, mantenerse al día con las lecturas, trabajos y actividades, realizar tareas administrativas, actividades de asistencia y actividades académicas en general. Las respuestas se expresan en una escala Likert de cinco puntos: nunca (1), casi nunca (2), a veces (3), casi siempre (4) y siempre (5).

Como variables independientes se consideraron sexo, edad, dedicación al estudio (exclusiva o compartida) y cuatro variables sobre gestión del tiempo, medidas a partir de las cuatro subescalas del Time Management Behavior Questionnaire (TMBQ, de Macan et al., 1990). De acuerdo con la revisión de Claessens et al. (2007), entre los cuestionarios habitualmente utilizados para medir la gestión del tiempo destacan tres instrumentos: Time Structure Questionnaire (TSQ), Time Management Questionnaire (TMQ) y Time Management Behavior Questionnaire (TMBQ), siendo este último el más utilizado en la investigación sobre este tópico. Para diseñar el TMBQ, sus autores se basaron en una lista de conductas de gestión del tiempo (Macan et al., 1990) por lo que fue construido para medir la amplitud o abundancia de conductas específicas indicadoras de manejo del tiempo, no la evaluación de los individuos sobre lo apropiadas o efectivas que son tales conductas. La prueba final consta de 34 ítems y utiliza una escala de respuesta tipo Likert de cinco puntos (nunca, pocas veces, algunas veces, habitualmente, siempre). De acuerdo con la estructura factorial del instrumento obtenida en el estudio original (Macan et al., 1990), los 34 ítems se agrupan en cuatro subescalas: Establecimiento de objetivos y prioridades, Herramientas para la gestión del 
tiempo, Percepción sobre el control del tiempo y Preferencias por la desorganización.

Una razón más de su utilización para este instrumento radica en la disponibilidad de una adaptación al español, validada empíricamente con alumnado universitario por García-Ros y Pérez-González (2012). Estos autores reprodujeron mediante análisis factorial exploratorio la estructura original de la prueba de cuatro factores, salvo movimientos o eliminación de algunos ítems. Las cuatro subescalas y sus indicadores psicométricos son los siguientes: Establecimiento de objetivos, que explica el $19.76 \%$ de la varianza y obtiene un alfa de Cronbach de 0.83; Herramientas para la gestión, que explica el $7.79 \%$ de la varianza y obtiene un alfa de Cronbach de 0.78; Preferencias en organización, que explica el $5.71 \%$ de la varianza con un coeficiente de confiabilidad de 0.71 y para Percepción de control del tiempo, que explica el $4.33 \%$ de la varianza, con un coeficiente de confiabilidad de 0.61. En cuanto a la fiabilidad de la escala en su conjunto, el coeficiente alfa de Cronbach fue de 0.83. Por otra parte, los resultados del estudio indican evidencia de validez convergente con otra prueba de gestión del tiempo (el TMQ) y evidencia de validez predictiva sobre el rendimiento académico.

Para su aplicación en Colombia, se realizó una validación lingüística de contenido y se identificaron algunos términos confusos, ambiguos o difíciles de entender, los cuales fueron sustituidos por vocablos o expresiones más próximos culturalmente al alumnado colombiano.

\section{Procedimiento}

Los instrumentos de recogida de datos se administraron mediante ordenador. Los estudiantes fueron reunidos en aulas de informática de sus respectivos centros, invitándoles a completar los instrumentos seleccionados. Los estudiantes participaron de manera voluntaria en el estudio, firmando un consentimiento previo a su participación. Los protocolos fueron aprobados por la Comisión de Ética e Investigación de la Universidad gestora del proyecto. Durante la aplicación, un investigador estuvo presente para resolver las inquietudes de los participantes. Junto a las respuestas a los instrumentos que miden procrastinación y gestión del tiempo, se recogieron los datos correspondientes a las variables demográficas sexo, edad y dedicación al estudio.

\section{Análisis}

Se ha partido de una descripción de las conductas procrastinadoras de los estudiantes, recurriendo al cálculo de medias y desviaciones estándar. Se valoró la normalidad de las variables a partir de su asimetría y curtosis. Tomando las seis variables que informan sobre la frecuencia en la demora de tareas, se realizó un análisis de clúster, utilizando como medida de disimilitud la distancia euclídea al cuadrado y como método de agregación el método de Ward. Este análisis permitió clasificar a los participantes en dos grupos, caracterizados respectivamente por exhibir niveles de menor o mayor procrastinación. Tras haber valorado la normalidad de las variables, la diferenciación entre ambos grupos fue contrastada mediante la prueba $\mathrm{t}$, acompañada de la $\mathrm{d}$ de Cohen para determinar la magnitud de las diferencias detectadas. Previamente, se aplicó la prueba de Levene para contrastar la homoscedasticidad de varianzas y seleccionar el estadístico $\mathrm{t}$ adecuado. Las puntuaciones medias en cada una de las subescalas del TMBQ fueron tomadas como variables independientes para llevar a cabo una regresión logística binaria, estableciendo como variable dependiente la pertenencia a los grupos de menor o mayor nivel de procrastinación. Con el fin de controlar sus efectos, en el modelo de regresión se incluyeron también como variables independientes aquellas características demográficas de los estudiantes que habían mostrado relaciones estadísticamente significativas con las conductas procrastinadoras de acuerdo con la prueba de chi-cuadrado, aplicable en este caso dado que se trata de variables categóricas. 


\section{Resultados}

El análisis de los factores de gestión del tiempo que explican las conductas procrastinadoras constituye el propósito central de este trabajo. La presentación de los resultados obtenidos en relación con este propósito irá precedida de una descripción de las variables implicadas y de la clasificación realizada para caracterizar al alumnado por sus niveles de procrastinación.

\section{Gestión del tiempo por el alumnado universitario}

Se partió de la estructura factorial encontrada para la adaptación al castellano del TMBQ, realizada por García-Ros y Pérez-González (2012), que a su vez resultaba muy similar a la del instrumento original desarrollado por Macan et al. (1990). Considerando las puntuaciones promedio de cada estudiante en los ítems que integran las respectivas subescalas, se calculó la media obtenida para cada uno de los factores (Tabla 1). La puntuación media en el total de ítems del TMBQ se sitúa en 3.34, por encima del punto medio de la escala (valor 3), lo cual indica una aceptable gestión del tiempo por parte del alumnado. Matizando esta afirmación, se aprecia un cierto déficit en la subescala referida al uso de Herramientas para la gestión del tiempo (2.89), claramente por debajo del resto de puntuaciones. Dado que para los ítems de carga negativa fue invertida la escala de puntuación, la media registrada para la subescala Preferencias por la desorganización (3.78) indica una baja preferencia de los estudiantes por el desorden y la desorganización. Para todas las variables se hallaron valores de asimetría y curtosis dentro del intervalo $[-1,1]$, lo cual permite considerar que las desviaciones distribucionales sobre la normalidad son poco importantes.
TABLA 1

Estadísticos descriptivos para la gestión del tiempo

\begin{tabular}{lllll}
\hline & Media & $\begin{array}{l}\text { Desviación } \\
\text { estándar }\end{array}$ & Asimetria & Curtosis \\
\hline Establecimiento de objetivos y prioridades & 3.46 & 0.63 & -0.619 & 0.501 \\
Herramientas para la gestión del tiempo & 2.89 & 0.87 & 0.168 & -0.629 \\
Percepción sobre el control del tiempo & 3.53 & 0.67 & -0.28 & -0.163 \\
Preferencias por la desorganización & 3.78 & 0.86 & -0.448 & -0.471 \\
Total de la escala TMB & 3.34 & 0.49 & 0.019 & -0.363 \\
\hline
\end{tabular}

Fuente: elaboración propia.

Niveles de procrastinación en el alumnado universitario

En el conjunto de la muestra de estudiantes analizada, las conductas procrastinadoras registran niveles medio-bajos (Tabla 2). En una escala de frecuencia que iba desde 1 (nunca) a 5 (siempre), las puntuaciones medias se ubican en valores ligeramente por debajo del punto central 3 (a veces). Con medias próximas a 2.7, destaca la frecuencia con que se posponen actividades tales como mantener al día el trabajo académico, escribir un trabajo final de curso o solicitar la asistencia del profesor. Por el contrario, las tareas administrativas son en las que menos se demora el alumnado universitario (media de 2.39). La asimetría y la curtosis en cada una de las variables no muestran valores elevados y resultan compatibles con una distribución aproximadamente normal

TABLA 2

Estadísticos descriptivos para las áreas de procrastinación

\begin{tabular}{lllll}
\hline & Media & $\begin{array}{l}\text { Desv. } \\
\text { Estándar }\end{array}$ & Asimetria & Curtosis \\
\hline Escribir un trabajo de final de curso & 2.69 & 0.84 & 0.188 & 0.311 \\
Estudiar para los exámenes & 2.54 & 0.97 & 0.417 & -0.118 \\
Mantenerme al dia con los trabajos / lecturas / actividades & 2.69 & 0.94 & 0.005 & -0.478 \\
$\begin{array}{l}\text { Tareas académicas administrativas: matricularse para las clases, } \\
\text { sacar el carnet, gestionar excusas, etc. }\end{array}$ & 1.1 & 0.423 & -0.623 \\
$\begin{array}{l}\text { Tareas de asistencia: pedir una cita con un profesor, pedir } 2.65 \\
\text { asesorias etc. }\end{array}$ & 1.11 & 0.24 & -0.712 \\
Actividades escolares en general & 2.51 & 0.93 & 0.332 & -0.035 \\
\hline
\end{tabular}

Fuente: elaboración propia.

Para caracterizar diferentes niveles de procrastinación en el alumnado, se efectuó un análisis clúster partiendo de las seis variables que informan sobre la frecuencia con que los estudiantes posponen diferentes tareas. Los valores en estas variables son el punto de partida para calcular la proximidad entre individuos, tomando como medida la distancia euclídea al 
cuadrado. La distancia euclídea es adecuada cuando, como en este caso, las variables son homogéneas y están medidas en unidades similares. Se ha adoptado un procedimiento de clasificación jerárquica, aplicando como procedimiento de agrupación el método de Ward, el cual permite optimizar la homogeneidad dentro de cada grupo y la separación entre grupos. El resultado de este análisis ha dado lugar a la clasificación que mostramos en el dendograma de la Figura 1, donde se aprecian dos grupos de sujetos unidos a una distancia reescalada de 25 , que integran a individuos o grupos de individuos unidos entre sí a distancias inferiores a 10. En el primer grupo se incluyen 240 estudiantes (media de 19.37 años; $58.2 \%$ son hombres; $59.91 \%$ con dedicación compartida), tamaño muy similar al del segundo grupo, constituido por 239 estudiantes (media de 19.26 años; $41.8 \%$ son hombres; $41.09 \%$ con dedicación compartida).

\section{Figura 1.}

Dendograma para la clasificación del alumnado según la frecuencia con que posponen tareas.

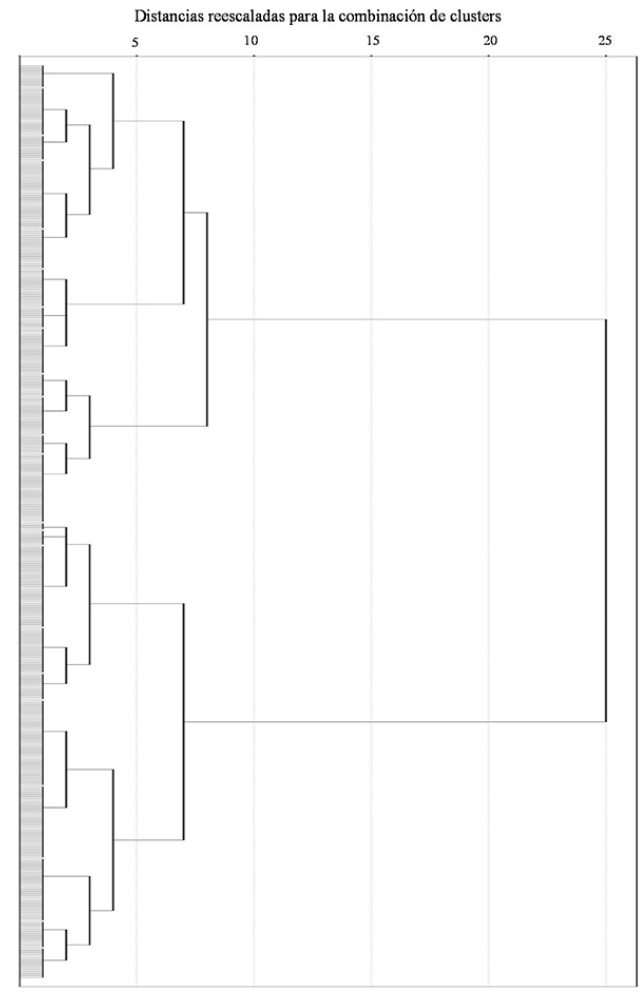

Fuente: elaboración propia.
Estos dos grupos de estudiantes presentan diferencias significativas en cuanto a la frecuencia con que posponen las tareas consideradas. Como muestra la Tabla 3, el primer grupo alcanza en todas ellas medias más altas que el segundo. Asumiendo el supuesto de normalidad y contrastando previamente la homoscedasticidad o igualdad de varianzas mediante la prueba de Levene, se calculó el estadístico $t$ para el contrate de medias. De acuerdo con el resultado obtenido, las diferencias observadas son estadísticamente significativas ( $p$ $=0)$, rechazando la hipótesis nula de que las medias son iguales y permitiendo afirmar que la frecuencia de las conductas procrastinadoras difiere en los dos grupos de alumnado. El tamaño del efecto se ha valorado mediante la d de Cohen, encontrándose diferencias entre grupos con una magnitud media (en torno a $d=0.5$ ) o grande (a partir de $d=0.8)$. En consecuencia, se pueden caracterizar ambos grupos como alumnado con distinto nivel de procrastinación, diferenciando los que poseen un nivel relativamente alto (grupo 1) frente a los que poseen un nivel bajo (grupo 2).

\section{TABLA 3}

Diferencias entre grupos resultantes de la clasificación del alumnado según frecuencia con que posponen tareas

\begin{tabular}{|c|c|c|c|c|}
\hline & $\begin{array}{l}\text { Medias } \\
\text { Grupo 1 } \\
(n=240)\end{array}$ & $\begin{array}{c}\text { Grupo 2 } \\
(n=239)\end{array}$ & Prueba $t$ & $\begin{array}{l}d \text { de } \\
\text { Cohen }\end{array}$ \\
\hline Escribir un trabajo de final de curso & 2.88 & 2.51 & $4.96^{*}$ & 0.45 \\
\hline Estudiar para los exámenes & 2.78 & 2.31 & $5.47^{\circ}$ & 0.5 \\
\hline Mantenerme al dia con los trabajos / lecturas / actividades & 3.02 & 2.36 & $8.38^{* \prime}$ & 0.77 \\
\hline $\begin{array}{l}\text { Tareas académicas administrativas: matricularse para las clases, } \\
\text { sacar el carnet, gestionar excusas, etc. }\end{array}$ & 2.97 & 1.81 & $13.61^{*}$ & 1.24 \\
\hline $\begin{array}{l}\text { Tareas de asistencia: pedir una cita con un profesor, pedir } \\
\text { asesorias etc. }\end{array}$ & 3.40 & 1.89 & $20.09^{*}$ & 1.84 \\
\hline Actividades escolares en general & 2.88 & 2.13 & $9.65^{*}$ & 0.88 \\
\hline
\end{tabular}

** La diferencia de medias es estadísticamente significatica con $p<0.001$. Fuente: elaboración propia.

Predicción de la procrastinación a partir de rasgos demográficos y gestión del tiempo

La regresión logística permite valorar la contribución de diferentes variables a la explicación de las conductas procrastinadoras en el alumnado universitario. Se ha tomado 
como variable dependiente binaria el nivel de procrastinación, construyendo un modelo para la predicción de las probabilidades de pertenecer al grupo de mayor nivel. Actúan como variables independientes categóricas las características demográficas de los estudiantes, cuya relación con el nivel de procrastinación ha resultado ser estadísticamente significativa al ser consideradas por separado: sexo $\left(\chi_{1}^{2}=4.288 ; p=0.038\right) \mathrm{y}$ dedicación $\left(\chi_{1}^{2}=16.454 ; p=0\right)$. En el caso de la edad, la correlación biserial puntual fue $\mathrm{rbp}=$ 0.016 , con $p>0.05$, por lo que esta variable fue excluida del análisis. Junto a sexo y dedicación incluimos como variables independientes en el modelo las puntuaciones promedio para cada una de las cuatro dimensiones identificadas en la gestión del tiempo. La varianza explicada por el conjunto de variables independientes, expresada mediante la $\mathrm{R}^{2}$ de Nagelkerke, asciende a 0.146. La prueba de Hosmer-Lemeshow arroja un valor chi cuadrado 7.603 ( $p=0.473)$, que lleva a mantener la hipótesis nula de ajuste del modelo a los datos observados. Otra medida de la bondad del modelo de regresión es su capacidad clasificatoria; se ha conseguido la clasificación correcta del $64.1 \%$ de los individuos.

La Tabla 4 muestra los valores estimados para los coeficientes del modelo, junto con el grado de significación p asociado al estadístico de Wald. En función de estos, se puede valorar el peso de cada variable en la explicación del nivel de procrastinación. En lo que respecta a los rasgos demográficos, el efecto de la dedicación $(\mathrm{B}=$ 0.654, $p=0.001)$ resulta significativo, mientras que la variable sexo $(B=0.133, p=0.579)$, que había mostrado una relación bivariable significativa con la procrastinación, pierde relevancia al ser considerada conjuntamente con las restantes variables incluidas en el modelo. Los factores de gestión del tiempo más importantes en la explicación de los niveles de procrastinación, con efectos negativos en ambos casos, son el Establecimiento de objetivos y prioridades $(\mathrm{B}=-0.554 ; \mathrm{p}=0.008)$, y la Percepción sobre el control del tiempo $(\mathrm{B}=$ $-0.547 ; p=0.001)$. Los dos restantes factores no contribuyen significativamente $(p>0.05)$ a explicar el nivel de procrastinación de los individuos. A partir de los coeficientes B se han calculado las odds ratio que expresan cuánto más probable es la pertenencia al grupo de mayor nivel de procrastinación cuando las variables predictoras incrementan su valor. La condición de estudiante con dedicación compartida $(\mathrm{OR}=$ 1.923) incrementa en un $92.3 \%$ la probabilidad de pertenecer al grupo de mayor nivel de procrastinación. En cambio, esta probabilidad se reduce a casi la mitad cuando aumenta en una unidad la puntuación en las dimensiones Establecimiento de objetivos y prioridades (OR $=0.575)$ o Percepción sobre el control del tiempo $(\mathrm{OR}=0.579)$.

\section{TABLA 4}

Regresión logística binaria para los efectos de los rasgos demográficos y la gestión del tiempo sobre la procrastinación

\begin{tabular}{llllll}
\hline & $\mathrm{B}$ & $\begin{array}{l}\text { Error } \\
\text { tipico }\end{array}$ & Wald & Sig. & $\begin{array}{l}\text { Odds } \\
\text { ratio }\end{array}$ \\
\hline Hombre $^{1}$ & 0.133 & 0.24 & 0.308 & 0.579 & 1.142 \\
Dedicación compartida $^{2}$ & 0.654 & 0.203 & 10.351 & 0.001 & 1.923 \\
Establecimiento de objetivos y prioridades & -0.554 & 0.209 & 7.023 & 0.008 & 0.575 \\
Herramientas para la gestión del tiempo & -0.082 & 0.143 & 0.327 & 0.568 & 0.921 \\
Percepción sobre el control del tiempo & -0.547 & 0.172 & 10.163 & 0.001 & 0.579 \\
Preferencias por la desorganización $_{\text {Constante }}$ & -0.058 & 0.134 & 0.189 & 0.664 & 0.943 \\
& 3.965 & 0.855 & 21.508 & 0 & 52.716 \\
\hline
\end{tabular}

1 Categoría de referencia: Mujer.

2 Categoría de referencia: Dedicación exclusiva. Fuente: elaboración propia.

\section{Discusión y conclusiones}

El presente trabajo ha contrastado el valor de la gestión del tiempo como variable predictora de la procrastinación en el alumnado universitario de primer año, contando para ello con una muestra amplia de estudiantes colombianos que han informado sobre la frecuencia con que posponen determinadas tareas y exhiben conductas de manejo del tiempo. Al estudiar el papel de las conductas de gestión del tiempo en la explicación de la procrastinación, también se tuvieron en cuenta características demográficas consideradas en los estudios sobre factores relacionados con la procrastinación (Rozental \& Carlbring, 2014; Steel, 2007). En presencia de estas, el Establecimiento de objetivos y prioridades, así como la Percepción 
sobre el control del tiempo han resultado ser buenos predictores de la procrastinación, mostrando con ella una relación negativa. Estos resultados confirman los obtenidos por Lay y Schouwenburg (1993), quienes hallaron una correlación inversa entre el TMBQ y la puntuación obtenida en un inventario de actividades académicas planificadas y cumplidas. La subescala de Establecimiento de objetivos parece reflejar de manera más adecuada elementos autorregulatorios que estarían en coherencia con la evaluación del déficit autorregulatorio que subyace a las conductas de procrastinación. Por otra parte, la percepción sobre el control del tiempo es una subescala reflejaría la percepción general que tiene la persona del manejo de su tiempo, derivada de su capacidad de planear y llevar adelante un manejo adecuado del mismo, conteniendo igualmente elementos de autorregulación que podrían estar presentes a la hora de evaluar un fallo autorregulatorio relacionado con las conductas de procrastinación.

Las evidencias encontradas en el presente estudio llevan a matizar la relación entre gestión del tiempo y procrastinación. Asumiendo el carácter multidimensional de las conductas de gestión del tiempo (García-Ros \& PérezGonzález, 2012; Macan et al., 1990), se constató que no todas las dimensiones tienen la misma importancia a la hora de explicar el nivel de procrastinación que exhiben los sujetos, resultando poco relevantes el uso de herramientas para la gestión del tiempo o la desorganización del trabajo y el desorden material en el lugar de estudio.

En cambio, los resultados del presente trabajo no ratifican el papel que estudios anteriores han atribuido a variables demográficas como sexo y edad en la explicación de la procrastinación académica (Rozental \& Carlbring, 2014; Steel, 2007). En este caso, no se ha hallado correlación entre edad y procrastinación. En cuanto al sexo, aunque significativamente se aprecia una mayor procrastinación en hombres que en mujeres, su efecto deja de ser relevante cuando se considera conjuntamente junto a las conductas de gestión del tiempo. Entre las variables demográficas, aquí se incluyó la condición de alumno con dedicación compartida que, en el caso del alumnado colombiano al igual que en otros países latinoamericanos, presenta una importante prevalencia. De hecho, en la muestra estudiada, el alumnado que simultanea estudios con trabajo se aproxima a la mitad. Esta variable ha resultado ser especialmente significativa en la predicción del nivel de procrastinación. Investigaciones previas de Forbus, Newbold y Mehta (2010) o Robotham (2012) llevaron a afirmar que la combinación de trabajo a tiempo parcial y estudios conducía al incremento de habilidades relacionadas con el adecuado manejo del tiempo, lo cual ayudaría a prevenir las conductas procrastinadoras. Sin embargo, los resultados obtenidos aquí no corroboran esa afirmación. Más bien al contrario, los estudiantes que combinan desempeño laboral con estudios corren el riesgo de procrastinar académicamente, probablemente debido a que buena parte del alumnado participante en el estudio no desempeñaría trabajos a tiempo parcial sino de jornada completa, lo que supone un importante obstáculo para su dedicación a los estudios.

La gestión del tiempo es una habilidad aprendida y, por tanto, susceptible de intervención, lo cual multiplica su importancia para prevenir la procrastinación, y también el fracaso y el abandono académico durante el primer año de ingreso a los estudios superiores. En efecto, diversos estudios aportan evidencia sobre la relación entre las habilidades de gestión del tiempo y el rendimiento académico (García-Ros \& Pérez-González, 2012; Gortner \& Zulauf 2000; Kitsantas, Winsler, \& Huie, 2008; Macan et al, 1990; Pehlivan, 2013). En su metaanálisis sobre los resultados de los principales programas de acompañamiento para estudiantes de primer año en las universidades norteamericanas Robbins, Oh, Le y Button (2009) encontraron que los programas centrados en habilidades académicas, incluyendo la gestión del tiempo, eran los más efectivos para conseguir una buena ejecución académica durante el primer año en la universidad. Por ello, son numerosas las intervenciones dirigidas 
específicamente al entrenamiento en estrategias de autorregulación, y en particular en habilidades para la gestión del tiempo, con la finalidad de prevenir la procrastinación y mejorar el rendimiento (Chen, 2011; Häfner, Oberst, \& Stock, 2014; Rosário et al., 2007; Schraw, Crippen, \& Hartley, 2006; Vovides, SanchezAlonso, Mitropoulou, \& Nickmans, 2007). Atendiendo a los resultados del presente estudio, este tipo de intervenciones habrían de hacer hincapié en los aspectos más relevantes de cara a prevenir la procrastinación relacionados con el aumento de las habilidades de autorregulación del aprendizaje: el establecimiento de objetivos y prioridades (por ejemplo, fijar metas a corto y largo plazo, evaluar la importancia de las tareas, priorizarlas, valorar los requerimientos en términos de esfuerzo y tiempo, fijar fechas límite, revisar el cumplimiento de objetivos) y la percepción del estudiante acerca del control que ejerce sobre su tiempo (p. ej., valorar el modo en que se gestiona el tiempo, tomar conciencia de cuándo se están aplazando tareas, perdiendo, malgastando u organizando inadecuadamente el tiempo disponible).

En el presente trabajo, se diferenciaron niveles de procrastinación con base en la frecuencia con que se posponen los seis tipos de tareas contemplados en el PASS, y no únicamente en la frecuencia de procrastinación ante el estudio de los exámenes, como propusieran inicialmente Rothblum, Solomon y Murakami (1986). Otra fortaleza de este trabajo se cifra en el tamaño y la heterogeneidad de la muestra empleada, que permite confiar en los resultados obtenidos. Entre las limitaciones del estudio, se encuentra el empleo de técnicas de autoinforme para obtener tanto los niveles de procrastinación del alumnado como sus conductas de gestión del tiempo, con el riesgo de que las respuestas de los estudiantes se vean afectadas por un sesgo de deseabilidad social. No obstante, el anonimato y la aclaración de los fines del estudio al administrar los instrumentos pueden haber contribuido a atenuarlo. Por otra parte, el planteamiento metodológico basado en un diseño correlacional impide llegar a conclusiones sobre relaciones causa-efecto, cuya comprobación requeriría el empleo de diseños experimentales o cuasi-experimentales. En esta línea podrían plantearse estudios futuros, dirigidos a valorar el efecto de la intervención sobre alumnado universitario de nuevo ingreso, centrando el tratamiento en el desarrollo de las capacidades para fijar y priorizar metas o en propiciar un control del tiempo por parte de los estudiantes. Este tipo de intervenciones, además, deberían dirigirse especialmente hacia el alumnado que simultanea estudios y trabajo, dado el mayor riesgo que corre este colectivo de incurrir en conductas procrastinadoras y, unido a ello, de protagonizar episodios de abandono o bajo rendimiento en sus estudios universitarios.

\section{Agradecimientos}

Se agradece a la Universidad El Bosque por la financiación y participación en este estudio y a La Fundación Universitaria Empresarial de la Cámara de Comercio de Bogotá (Uniempresarial) por su participación.

\section{Referencias}

Barber, L., Munz, D., Bagsby, P., \& Grawitch, M. (2009). When does time perspective matter? Self-control as a moderator between time perspective and academic achievement. Personality and Individual Differences, 46, 250-253. http:// dx.doi.org/10.1016/j.paid.2008.10.007

Bogg, T., \& Roberts, B. W. (2004). Conscientiousness and health-related behaviors: A meta-analysis of the leading behavioral contributors to mortality. Psychological Bulletin, 130, 887-919. http:// dx.doi.org/10.1037/0033-2909.130.6.887

Cardona, L. (2015). Relaciones entre procrastinación académica y estrés académico en estudiantes universitarios (Tesis de pregrado). Universidad de Antioquia, Medellín, Colombia. Recuperado de http:// 200.24.17.74:8080/jspui/handle/fcsh/242

Carranza, R., \& Ramírez, A. (2013). Procrastinación y características 
demográficas asociados en estudiantes universitarios. Revista Apuntes Universitarios, 3(2), 95-108.

Chen, P. (2011). Guiding college students to develop academic self-regulatory skills. Journal of College Teaching and Learning, 8(9), 29-33.

Claessens, B., Van Eerde, V., Rutte, Ch., \& Roe, R. (2007). A review of the time management literature. Personnel Review, 36(2), 255-276. http:// dx.doi.org/10.1108/00483480710726136

Dura\#n-Aponte, E., \& Pujol, L. (junio, 2012). Estilos de aprendizaje, gestión del tiempo y rendimiento académico en estudiantes universitarios. Trabajo presentado en el V Congreso Mundial de Estilos de Aprendizaje Santander, España. Recuperado de https://dialnet.unirioja.es/d escarga/articulo/4635078.pdf

Dura\#n-Aponte, E. \& Pujol, L. (2013). Manejo del tiempo acade\#mico en jo\#venes que inician estudios en la Universidad Simo\#n Boli\#var. Revista Latinoamericana de Ciencias Sociales, Niñez y Juventud, 11(1), 93-108. http:// dx.doi.org/10.11600/1692715x.1115080812

Ferrari, J. R. (1993). Christmas and procrastination: Explaining lack of diligence at a "real-world" task deadline. Personality and Individual Differences, 14, 25-33. http:// dx.doi.org/10.1016/0191-8869(93)90171. $\mathrm{X}$

Ferrari, J. R., Mason, C., \& Hammer, C. (2006). Procrastination as a predictor of task perceptions: Examining delayed and non-delayed tasks across varied deadlines. Individual Differences Research, 4(1), 28-36.

Ferrari, J. R., O'Callaghan, J., \& Newbegin, I. (2005). Prevalence of procrastination in the United States, United Kingdom, and Australia: Arousal and avoidance delays among adults. North American Journal of Psychology, 7(1), 1-6.

Forbus, P., Newbold, J., \& Mehta, S. (2010). A study of non traditional and traditional students in terms of their time management behaviors, stress factors, and coping strategies. Proceedings of the Academy of Educational Leadership, 15(2), 67-71.

Garzón, A., \& Gil, J. (2017a). El papel de la procrastinación académica como factor de la deserción universitaria. Revista Complutense de Educación, 28(1), 307-324. http://dx.doi.org/10.5209/ rev_RCED.2017.v28.n1.49682

Garzón, A., \& Gil, J. (2017b). Propiedades psicométricas de la versión en español de la prueba PASS. Revista Iberoamericana de Diagno\#stico y Evaluacio\#n Psicológica, 1(43), 149-163. http://dx.doi.org/10.21865/RIDEP43_149

García-Ros, R., \& Pérez-González, F. (2012). Spanish version of the Time Management Behavior Questionnaire for University Students. The Spanish Journal of Psychology, 15(3), 1485-1494. http://dx.doi.org/10.5209/ rev SJOP.2012.v15.n3.39432

George, D., Dixon, S., Stansal, E., Lund, S., \& Pheri, T. (2008). Time diary and questionnaire assessment of factors associated with academic and personal success among university undergraduates. Journal of American College Health, 56(6), 706-715. http://dx.doi.org/10.3200/ JACH.56.6.706-715

Gortner, A., \& Zulauf, C. (2000). Factors associated with academic time use and academic performance of college students: A recursive approach. Journal of College Student Development, 41 (5), 544-556.

Häfner, A., Oberst, V., \& Stock, A. (2014). Avoiding procrastination through time management: An experimental intervention study. Educational Studies, 40(3), 352-360. http:// dx.doi.org/10.1080/03055698.2014.899487

Harriott, J. S., \& Ferrari, J. R. (1996). Prevalence of procrastination among samples of adults. Psychological Reports, 78, 611-616. http:// dx.doi.org/10.2466/pr0.1996.78.2.611

Kaya, H., Kaya, N., Ozturk, A., \& Kucuk, L. (2012). Assessing time-management skills in terms of age, gender, and anxiety levels: 
A study on nursing and midwifery students in Turkey. Nurse Education in Practice, 12, 284-288. http://dx.doi.org/10.1016/ j.nepr.2012.06.002

Kim, K. R., \& Seo, E. H. (2015). The relationship between procrastination and academic performance: A meta-analysis. Personality and Individual Differences, 82, 26-33. http:// dx.doi.org/10.1016/j.paid.2015.02.038

Kitsantas, A., Winsler, A., \& Huie, F. (2008). Self-regulation and ability predictors of academic success during college: A predictive validity study. Journal of Advanced Academics, 20(1), 42-68.

Klingsieck, K. B. (2013). Procrastination: When good things don't come to those who wait. European Psychologist, 18, 24-34. http:// dx.doi.org/10.1027/1016-9040/a000138

Krumrei, M., Elizabeth, J., Newton, F, Kim, E., \& Wilcox, D. (2013). Psychosocial factors predicting first-year college student success. Journal of College Student Development, 54(3), 247-266.

Lay, C. H. (1990). Working to schedule on personal projects: An assessment of person-project characteristics and trait procrastination. Journal of Social Behavior and Personality, 5, 91-103.

Lay, C. H., \& Burns, P. (1991). Intentions and behavior in studying for an examination: The role of trait procrastination and its interaction with optimism. Journal of Social Behavior and Personality, 6, 605-617.

Lay, C., \& Schouwenburg, H. (1993). Trait procrastination, time management, and academic behavior. Journal of Social Behavior 8 Personality, 8(4), 647-662.

Liu, L., Rijmen, F., MacCann, C., \& Roberts, R. (2009). The assessment of time management in middle-school students. Personality and Individual Differences, 47, 174-179. http://dx.doi.org/1016/ j.paid.2009.02.018

Macan, T., Shahani, C., Dipboye, R. L., \& Phillips, A. P. (1990). College students' time management: Correlations with academic performance and stress. Journal of Educational Psychology, 82 (4), 760-768.
Olowookere, E., Alao, A., Odukoya, J., Adekeye, O., \& Ade' Agbude, G. (2015). Time management practices, character development and academic performance among university undergraduates. Creative Education, 6, 79-86. http:// dx.doi.org/10.4236/ce.2015.61007

Pehlivan, A. (2013). The effect of the time management skills of students taking financial accounting course on their course grades and grade point averages. International Journal of Business and Social Science, 4(5), 196-199.

Pintrich, P. R., Smith, D., García, T., \& McKeachie, W. (1993). Reliability and predictive validity of the Motivated Strategies for Learning Questionnaire (MSLQ). Educational and Psychological Measurement, 53, 801-813. http:// dx.doi.org/10.1177/0013164493053003024

Pychyl, T. A., \& Flett, G. L. (2012). Procrastination and self-regulatory failure: An introduction to the special issue. Journal of Rational-Emotive $\mathcal{B}$ CognitiveBehavior Therapy, 30, 203-212. http:// dx.doi.org/10.1007/s10942-012-0149-5

Robbins, S., Oh, I., Le, H., \& Button, C. (2009). Intervention effects on college performance and retention as mediated by motivational, emotional, and social control factors: Integrated meta-analytic path analyses. Journal of Applied Psychology, 94(5), 1163-1184. http://dx.doi.org/10.1037/a0015738

Robotham, D. (2012). Student parttime employment: Characteristics and consequences. Education + Training, 54(1), 65-75. http:// dx.doi.org/10.1108/00400911211198904

Rosário, P., Mourão, R., Núñez, J. C., GonzálezPienda, J., Solano, P., \& Valle, A. (2007). Eficacia de un programa instruccional para la mejora de procesos y estrategias de aprendizaje en la enseñanza superior. Psicothema, 19(3), 422-427.

Rothblum, E., Solomon, L., \& Murakami, J. (1986). Affective, cognitive, and behavioral differences between high and 
low procrastinators. Journal of Counseling Psychology, 33(4), 387-394.

Rozental, A., \& Carlbring, P. (2014). Understanding and treating procrastination: A review of a common self-regulatory failure. Psychology, 5, 1488-1502. http://dx.doi.org/10.4236/ psych.2014.513160

Schraw, G., Crippen, K., \& Hartley, K. (2006). Promoting self-regulation in science education: Metacognition as part of a broader perspective on learning. Research in Science Education, 36, 111-139. http:// dx.doi.org/10.1007/s11165-005-3917-8

Solomon, L., \& Rothblum, E. (1984). Academic procrastination: Frequency and cognitivebehavioral correlates. Journal of Counseling Psychology, 31 (4), 503-509.

Steel, P. (2007). The nature of procrastination: A meta-analytic and theoretical review of quintessential self-regulatory failure. Psychological Bulletin, 133(1), 65-94. http:// dx.doi.org/10.1037/0033-2909.133.1.65

Tuckman, B.W. (2003). The effect of learning and motivation strategies training on college students' achievement. Journal of College Student Development, 44(3), 430-437. http://dx.doi.org/10.1353/ csd.2003.0034

Van Eerde, W. (2003). A metaanalytically derived nomological network of procrastination. Personality and Individual Differences, 35, 1401-1418. http://dx.doi.org/10.1016/ S0191-8869(02)00358-6

Vovides, Y., Sanchez-Alonso, S., Mitropoulou, V., \& Nickmans, G. (2007). The use of e-learning course management systems to support learning strategies and to improve self-regulated learning. Educational Research Review, 2, 64-74. http://dx.doi.org/10.1016/ j.edurev.2007.02.004

Zimmerman, B. (1996). Enhancing student academic and health functioning: A self regulatory perspective. School Psychology Quarterly, 11(1), 47-66. http:// dx.doi.org/10.1037/h0088920

\section{Notas}

* Artículo de investigación. 